\title{
Intellectual disability-balding-patella luxation-acromicria syndrome
}

INSERM

\section{Source}

INSERM. (1999). Orphanet: an online rare disease and orphan drug data base. Intellectual disability-balding-patella luxation-acromicria syndrome. ORPHA:3041

Intellectual disability-balding-patella luxation-acromicria syndrome is characterised by severe intellectual deficit, patella luxations, acromicria, hypogonadism, facial dysmorphism (including midface hypoplasia and premature frontotemporal balding). It has been described in three unrelated males. 